Review

\title{
VNARs: An Ancient and Unique Repertoire of Molecules That Deliver Small, Soluble, Stable and High Affinity Binders of Proteins
}

\section{Caroline Barelle * and Andy Porter}

Elasmogen Ltd, Scottish Biologics Facility, Foresterhill, Aberdeen, AB25 2ZP, UK;

E-Mail: a.porter@abdn.ac.uk

* Author to whom correspondence should be addressed; E-Mail: caroline.barelle@elasmogen.com; Tel.: +1-1224-438545.

Academic Editor: Friedrich Koch-Nolte

Received: 21 July 2015 / Accepted: 18 August 2015 / Published: 1 September 2015

\begin{abstract}
At 420 million years, the variable domain of New Antigen Receptors or VNARs are undoubtedly the oldest (and smallest) antigen binding single domains identified in the vertebrate kingdom. Their role as an integral part of the adaptive immune system of sharks has been well established and has served to provide a greater understanding of the evolution of humoral immunity; their cellular components and processes as well as the underlying genetic organization and molecular control mechanisms. Intriguingly, unlike the variable domain of the camelid heavy chain antibodies or VHH, VNARs do not conform to all of the characteristic properties of classical antibodies with an ancestral origin that clearly distinguishes them from true immunoglobulin antibodies. However, this uniqueness of their origin only adds to their potential as next generation therapeutic biologics with their structural and functional attributes and commercial freedom all enhancing their profile and current success. In fact their small size, remarkable stability, molecular flexibility and solubility, together with their high affinity and selectivity for target, all reinforce the potential of these domains as drug candidates. The purpose of this review is to provide an overview of the existing basic biology of these unique domains, to highlight the drug-like properties of VNARs and describe current progress in their journey towards the clinic.
\end{abstract}

Keywords: VNAR; biologics; antibody; single-domain; shark; therapeutics; humanization; stability; shark 


\section{Introduction: Their Basic Beginnings}

\subsection{Adapting to Attack}

Early studies conducted in the 1960s provided the first evidence that sharks could elicit a protective response against antigen challenge [1]. This work supported the existence of an adaptive immune system in these ancient vertebrates. However, it took another three decades before the molecular and cellular foundation of this humoral response began to be properly elucidated. As with all basic biological research many questions still remain to be fully answered but great steps have been taken towards our understanding of the mechanisms underpinning immunity in these incredible and ancient animals.

Sharks belong to the class Chondrichthyes or Cartilaginous fish which can be divided into two subclasses: the elasmobranchs (sharks, rays and skates) and the holocephalans (chimaeras and ratfish). Classification was originally based on a combination of gross anatomical features including characteristics such as gill slit number. With the advent and refinement offered by molecular characterization a greater understanding of the relationship between species now exists across the eight distinct orders of elasmobranchs [2]. Estimates of the evolutionary age of these ancient fish, places them in the Ordovician period, a staggering 450 million years ago, rendering them the oldest vertebrate taxon to possess an adaptive immune system. This more comprehensive picture now confirms the presence not only of immune players such as immunoglobulins, T cells and major histocompatibility complex (MHC) but also of processes such as somatic hypermutation, recombinase-activating gene (RAG) activity and sites of intense cellular activity including the spleen [3,4]. A point that is often overlooked, is that sharks are all cartilaginous species, and therefore do not have any semblance of bone marrow. However, they do possess unique specialized tissues attached to the gonads (epigonal) and in some species, the esophagus (Leydig) where it is believed lymphopoiesis occurs. Supporting this hypothesis is the presence of developing granulocytes, blast cells, and plasma cells, together with expression of RAG recombinases, TdTs and B cell specific transcription factors consistent with their role in lymphocyte development [5-7]. The epigonal organ in particular is the site of secreting B cells in adult animals confirming its function as an Elasmobranchii equivalent of mammalian bone marrow [8]. Secondary lymphoid tissues have also been identified in sharks including a spleen and thymus. Although true germinal centres have not been identified, regions of cellular differentiation in the spleen (forming white and red pulp zones) have been detected and associated with B cell activation and maturation processes [8].

Of the immunoglobulins, $\operatorname{IgM}$ was the first to be identified from immunization studies conducted in the 1960s through to the 1980s. Two distinct serum fractions of immune responsive, 19S and 7S, were isolated representing the pentameric and monomeric forms of IgM respectively. Insight into the temporal expression of these antibody subtypes was also reported showing an initial enrichment of 19S followed by $7 \mathrm{~S}$. Some of this early work also suggested a secondary enrichment of $19 \mathrm{~S}$. Taken together this was the first evidence that sharks had the capacity to respond to foreign challenge in a coordinated antibodydependent fashion [9-14]. In line with classical B cell receptor systems, shark IgM exists in both transmembrane (for activation of lymphocytes) and secretory forms (to bind antigen and induce effector functions of the humoral system) [15]. More detailed studies in nurse shark revealed multiple forms of this Ig isotype, generated through alternative splicing. Three secretory forms of IgM have been identified that include the aforementioned $19 \mathrm{~S}$ pentameric form and the $7 \mathrm{~S}$ monomeric form, composed of five 
immunoglobulin superfamily (Igsf) domains. An additional, four domain, germline-joined secretory IgM (IgM $\mathrm{Igj}_{\mathrm{g}}$ ) was also identified in nurse shark. As the expression profile of this variant showed a high level at birth, it is assumed that it functions in early development $[6,16]$. For nurse shark at least, a five domain transmembrane form completes the IgM heavy chain family [15].

The function of the second conventional Ig member of the antibody repertoire of sharks known as $\mathrm{IgW}$ remains somewhat of an enigma. As with the IgM isotype, the most studied IgW heavy chain family is that of the nurse shark, revealing the long and short subtypes consisting of seven and three Igsf domains respectively. Both shorter forms exist as secretory and transmembrane bound variants whereas the long, seven domain form is only secretory, with no identified transmembrane counterpart. The fourth and final member of this heavy chain group is a second transmembrane bound form consisting of five domains. Expression levels of each type of $\operatorname{IgW}$ varied in individual animals, perhaps indicative of varying exposure to a pathogen or antigen class, but no definitive pattern has been reported. Interestingly, levels of the short secretory and transmembrane form were also isolated from the pancreas suggesting the possibility of a GALT-type (gut associated lymphoid tissue) response in these animals [15].

The final immunoglobulin member of this humoral tool box and indeed the protagonist of this of this review is the New Antigen Receptor or IgNAR. First identified in 1995 by Andrew Greenberg in nurse sharks (Ginglymostoma cirratum), the original Ig-based nomenclature stems from the initial belief that it constituted a related B Cell Receptor (BCR) to classical immunoglobulins [17]. In many respects, IgNAR does fit the profile of a true antibody as it is both structurally similar and follows analogous target-specific kinetics of expression in response to immunogen challenge. However further scrutiny of this adaptive molecule has afforded a greater understanding of the evolutionary origin of IgNAR and whilst its role is similar, its ancestral origin is very different from that of a classical antibody.

\subsection{To be or Not to Be an Antibody}

In the case of IgNAR current thinking would likely generate an answer that is "maybe yes" but more probably "no". To explain this apparent dichotomy demands a more thorough analysis of this unique molecule.

IgNAR exists naturally in the sera of sharks as a covalently linked heavy chain homodimer at circulating concentrations estimated to be between $0.1-1.0 \mathrm{mg} / \mathrm{mL}[17,18]$. The lack of a light chain partner (now and throughout its history) is the first of many distinctions separating this molecule from its classical adaptive antibody counterparts including $\operatorname{IgM}$ and $\operatorname{IgW}$. IgNAR exists, in common with classical B cell receptors, as a soluble and receptor bound form consisting of a variable domain known as VNAR, with differing numbers of constant domains (Figure 1). Two distinct splice variants of the receptor bound form exist, with three and five constant domains, compared with a single soluble type composed always of five constant domains [15,17]. Kinetic analyses of the constant domains revealed the ability of $\mathrm{C} 1$ and $\mathrm{C} 3$ to independently dimerise with dissociation constants of $595 \pm 43 \mathrm{nM}$ and $188 \pm 16 \mu \mathrm{M}$ respectively suggesting that $\mathrm{C} 1$ drives the dimerization process. Structural analysis of these domains revealed a wide angle at the $\mathrm{C} 1$ dimer interface creating a considerable level of flexibility and distance between the variable domains presumably enabling or facilitating antigen interaction [19]. 

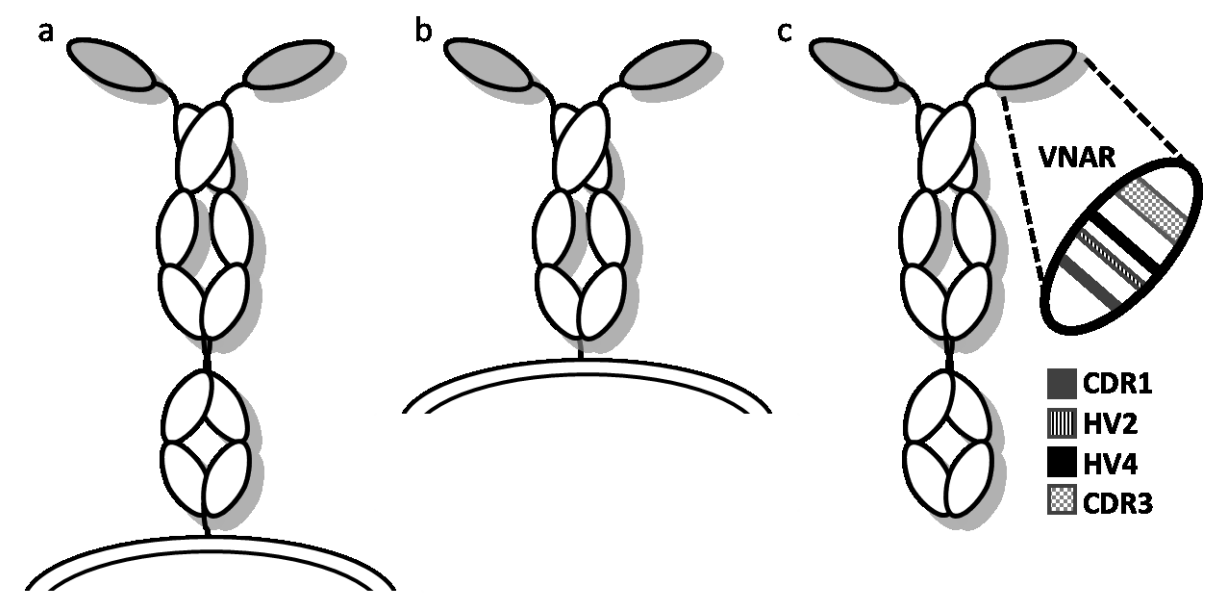

Figure 1. Cartoon depicting the different forms of IgNAR in nurse shark. (a) Six domain membrane bound homodimer; (b) Four domain membrane bound homodimer; (c) Six domain secreted form of IgNAR. Variable (VNAR) domains are shown as shaded ovals; constant domains are white ovals.

Its role in adaptive immunity was first demonstrated in the labs of Flajnik and Porter where hen egg lysozyme (HEL) was used as a model antigen to immunise nurse sharks. At this time, no tool reagents existed to monitor any antigen-specific titres so a phagemid library of amplified VNAR domains was built after several months of iterative protein boosts and the monitoring of maturation at the DNA level. This library yielded a number of hits, the lead clone being designated 5A7 with an affinity for HEL of $20 \mathrm{nM}$ as a soluble monomeric domain [20]. This work was continued through hyperimmunisation of the same animals. A rapid, increased response was demonstrated confirming both specificity and memory and thereby verifying the role of IgNAR as a component of adaptive immunity [21]. In addition to this clear early evidence of VNAR domains undergoing in vivo maturation, a more detailed analysis of this process exposed differences as well as similarities from that seen in mammalian antibody maturation. In sharks, features of classical somatic maturation are present and include: strand bias and serine codon hotspots. However, in stark contrast to classical antibody maturation, base changes tend to occur in tandem as doublets or triplets and as palindromic repeats [22]. In addition, the expression of a low affinity, high avidity pentameric IgM expression pattern was detected early (mirroring higher vertebrates) followed by an antigen-specific monomeric IgM response. This IgM antibody response was initiated well before any measurable IgNAR response.

IgNAR has been identified in a number of different shark species such as smooth dogfish (Mustelus canis), spotted catfish (Scyliorhimus canicula), wobbegong (Orectolobus maculatus), banded houndshark (Triakis scyllium) and bamboo shark (Chiloscyllium plagiosum) [23-30]. However evidence of an IgNAR based adaptive response has yet to be demonstrated in these animals. We would propose that such a response to challenge is highly likely based on the existing evidence by Müller et al. who successfully showed that spiny dogfish (Squalus acanthias) can elicit an antigen-specific IgNAR response after immunization [31,32]. The significance of this work was not only to develop a new animal model for IgNAR generation but evolutionarily implied the existence of IgNAR prior to the point of 
divergence of sharks and the batoids (rays and skates) approximately 220 million years ago; supporting the idea that IgNAR existed in an earlier ancestral organism now continued through the Elasmobranch lineage. To date, the greatest understanding of the IgNAR immune response has emanated from studies conducted in nurse shark but immunization of horn shark has also yielded IgNAR target specific responses [20,33].

Structurally, VNAR domains have a classic immunoglobulin fold and superimposition of human variable heavy and light chains onto VNAR domains, revealed a structural relatedness within the core framework [34]. However this remarkable example of structural convergent evolution, is where the similarity ends as sequence comparison reveals only $25 \%-30 \%$ identity to mammalian heavy chains. In fact, a nominally greater percentage identity is seen between light chains and $\mathrm{T}$ cell receptors than to antibody heavy chains [35]. It is this T cell similarity from which the nomenclature for the two extra loops on the VNAR domain was coined - namely HV2 and HV4. Critically these occupy a region within the domain which would normally be occupied by CDR2 and would typically function to stabilize the interface with the light chain partner in a classical antibody. Subsequently a greater percentage of polar or charged residues are found in VNAR domains across this region compared to the conserved hydrophobic residues found at the classical VH-VL interface [36].

A further clue to the evolutionary origin of the VNAR domain was uncovered through the work of Criscitiello and colleagues who demonstrated the existence of a VNAR TCR fusion [37]. This makes VNAR the first V domain to be integral to the biology of both Ig and TcR. It is this unique organization of the cartilaginous antigen receptor genes that permits this combinatorial plasticity. Unlike the translocon gene system found in other vertebrates, the Ig genes (IgNAR, IgW and $\operatorname{IgM}$ ) of cartilaginous fish are organized into clusters each consisting of one $\mathrm{V}$ segment, one or more D segments, one J segment and the constant domain exons [38,39]. Hundreds of clusters can exist in one species. Such gene organization negates class switching, which is well established in other vertebrates, but has enabled evolutionary recombination events such as those demonstrated between the NAR V gene and TRD. This genetic mobility has also occurred historically between the Ig clusters of IgW and IgNAR, exemplified by the homology of the last four constant domains in IgNAR with those in IgW and appears indicative of a common ancestor $[15,16,40]$. Until recently, these data placed the origins of VNAR to a point at least 220 million years ago, however with the recent completion of a genome sequencing project, this timeline has been moved backwards to 420 million years. The genome in question was that of a holocephalon, C. milii, more commonly known as the elephant shark. Assembly of the sequence of this ancient vertebrate revealed no evidence of IgNAR or IgW, but did uncover the presence of NAR-TCR. This would strongly suggest that a $\mathrm{T}$ cell receptor fusion pre-dates the recombination of VNAR with an Ig locus and places that event at the point in time when the chondrichthyan split into the holocephalan and elasmobranch lineages [41]. VNAR therefore did not originate as an Ig but was co-opted during evolution probably afforded by the molecular emphasis on cluster gene arrangement.

One other class of animals is known to produce a binding protein that shows significant structural similarities to shark VNAR. Camelidea (camel, llamas, etc.) VHH domains are small, soluble and have a pre-disposition to bind into pockets and clefts of antigens. Unlike VNAR, VHH binding sites are derived from a true antibody lineage and are believed to have originated from a typical IgG molecule that underwent period of selection and the permanent loss of its light chain resulting in a prolate shaped (rugby-ball) binding site that recognizes epitopes or antigen classes not well served by more planar 
(6 loop) binding sites consisting of both a VH and VL domain [42]. Here again is a related but equally striking example of convergent evolution with two Ig molecules, one clearly an antibody (VHH) and a second (VNAR) that is just as clearly not an antibody (never has had or lost a light chain), where both have under gone a period of selection pressure to evolve a binding site paratope capable of recognizing epitopes poorly served by the other components of their respective immune systems $[42,43]$.

Commercially it is worth noting that this evolutionary separation of VNAR from classical antibodies affords significant intellectual property (IP) benefits as it places them outside of the complex and crowded antibody patent landscape. Such broad freedom-to-operate should accelerate the development of therapeutic VNAR domains as the basis of biologic drugs that can recognize and neutralize both validated and novel protein targets.

\section{Exploiting the Benefits of VNAR}

\subsection{Biologics Are Leading the Way}

With total revenues in excess of $\$ 150$ billion and continued strong market growth, biologics have taken the pharmaceutical industry by storm. Rather than resting on their laurels, protein drug developers have continued to innovate. Improvements in expression and purification systems, coupled with increased knowledge at the molecular level, are enabling tailored pharmacokinetic and biophysical properties through customized reformatting. Taken together this has resulted in enhanced potency and more importantly improvements in clinical efficacy. Within this sector, antibodies are top of the leader board with half the top ten blockbuster drugs (annual revenues of greater than $\$ 1$ billion) derived from mammalian mAbs (monoclonal antibodies). The success of mAbs can possibly be distilled down to a few key inherent characteristics; (1) exquisite specificity; (2) high affinity; (3) long serum half-life; (4) amenability to molecular engineering. In addition to these drug-like attributes, enabling technologies have played a significant role in the last few decades and include combinatorial selection technologies such phage display which has provided a powerful platform for isolation of fully human antibodies from libraries of almost 1 trillion different clones.

Despite delivering excellent results in a range of clinical indications many in the industry believe that the use of mAbs should be considered just the first phase of this therapeutic revolution and point to well recognized limitations of these molecules including their large size, complex composition and relatively fragile nature; problems which could be tackled by the development of "next-generation" biologic therapies. Both antibody-related and novel scaffolds are being progressed towards the clinic in the hope that these new formats will overcome the shortfalls of mAbs, and improve patient outcomes [44].

Foremost in the design of next generation biologics is a desire to retain the positive attributes of mAbs whilst simplifying the approach to avoid complex and cumbersome downstream drug-development bottle-necks, allowing the addition of novel features such as war-head delivery for cell killing or the recognition of multiple drug targets. In general terms what is being asked of next-generation of biologic drugs is improved therapeutic potency but from less protein. 


\subsection{VNAR as a Candidate Therapeutic Domain}

To determine the viability of these antibody-like domains as candidate therapeutics, a number of criteria must be fulfilled. Basics such as specificity, stability and manufacturability are all essential attributes for any new protein drug-discovery approach. However, in this hugely competitive marketplace these basic requirements are simply not enough. A new biological drug class must differentiate from and deliver more than existing "gold-standard" approaches. The purpose of the following sections is to review the evidence to date verifying the attributes of VNAR domains and determine their potential to become effective therapeutic drugs.

\subsubsection{Naturally Adapted for Drug Development}

IgNAR is an integral part of the adaptive immune system of sharks. Critically this provides VNAR with an inherent advantage over other novel scaffolds as it naturally binds target with high affinity and selectivity. Immunization is a powerful means of inducing a target specific antibody response and has been the mainstay of therapeutic antibody development for decades. Standard model animals such as rabbits and mice have fueled the production of effective reagent and therapeutic antibodies. In addition, immunized camels and llamas have been the source of several therapeutic VHH (nanobodies) and are reviewed in this Special Edition. The power of immunization as a route to binders lies in the process of in vivo or somatic hypermutation. The challenge when immunizing a mammalian species is eliciting an immune response against conserved mammalian proteins; often important drug targets for biologics therapy. To overcome this problem, processes to break tolerance are frequently employed. This is simply not the case with sharks. Being phylogenetically distinct from all mammals avoids this problem completely and enables strong adaptive responses to be achieved against highly conserved mammalian proteins. The highest affinity VNAR domain isolated to date, known as E06, was isolated from a spiny dogfish immunized with human serum albumin (HSA). As a monomer (no avidity effect) this domain has an affinity of $200 \mathrm{pM}$. This result not only exemplifies the power of somatic mutation to generate high affinity binders but also the concept of raising in sharks a response against a conserved human protein. As albumin is absent from shark serum the animals elicited a powerful immune response to this "very foreign" antigen [31].

\subsubsection{Small Size but Large Diversity}

At approximately $12 \mathrm{kDa}$, VNAR domains are the smallest naturally occurring independent binding domains in the vertebrate kingdom, and yet they are capable of binding to targets with high affinity and exquisite selectivity [17]. This ability is delivered from very large repertoire diversity achieved through antigen driven maturation of primarily four hypervariable loops (CDR1, CDR3 HV2 and HV4). This differs from the three loops seen in a conventional antibody domain (including $\mathrm{VHH}$ ) and translates into structural features that enhance the interface between the VNAR and its target. To fully appreciate the binding site benefits of this unusual 4 loop, paratope topology, requires an understanding of the different types of IgNAR; their similarities and differences.

The antibody-like qualities of these domains with their functional and structural similarities to classical BCRs fueled further research to understand their basic biology and origins of this unique 
immune molecule. Whilst research was driven by their functional similarities to antibodies, the first sequence information generated for VNAR clearly illustrated the profound differences between these elasmobranch binding domains and mammalian Igs [17]. In particular this analysis highlighted the absence of a light chain partner and CDR2 and the presence of HV2 and 4 loops.

In depth descriptions of the different types of VNAR domains have been reviewed recently however for completeness, Figure 2 illustrates the main family members [44,45]. Unlike classical VH domains which consist of nine beta strands, VNAR domains are smaller due to the absence of $\mathrm{C}^{\prime}$ and $\mathrm{C}^{\prime \prime}$ strands [45-47]. All types contain the same canonical cysteine residues that act to hold this Igsf core structure. The key differences arise from the presence of non-canonical cysteines in both CDR loops and frameworks designated as stars in Figure 2.

Research conducted primarily on two species of shark (nurse and wobbegong) provided the first crystal structures of VNAR domains in complex with their cognate antigens, HEL and AMA-1 respectively $[46,47]$. These studies demonstrated beautifully the structural features of the additional non-canonical cysteine residues in both Type I and Type II domains. The resultant disulphide bridges cross the CDR and framework regions to form finger-like protrusions that create an extended convex paratope which can bind deep into protein clefts on the target molecule ("canyon-binders"). The constraints of the disulphide bridges provide the necessary stability to support the extended CDR3 loops. In the case of Type I VNAR domains these can be lengths of up to 35 residues long [21]. Both VNAR and VHH exhibit these disulphide stabilized antigen binding loops and it has been hypothesized that exposure to harsh physiological conditions is the driving force behind these structural features [43]. In the case of sharks, their blood contains high urea and salt creating a challenging environment for a protein binding domains [21]. An interesting and valuable consequence of these protruding paratopes is the ability of VNAR domains to target and bind to cryptic or hidden epitopes that elude the more concave classical antibody antigen interfaces. This is evident from the crystal structures of the HEL binding Type I and Type II VNAR domains but has since been demonstrated against a number of therapeutically relevant targets.

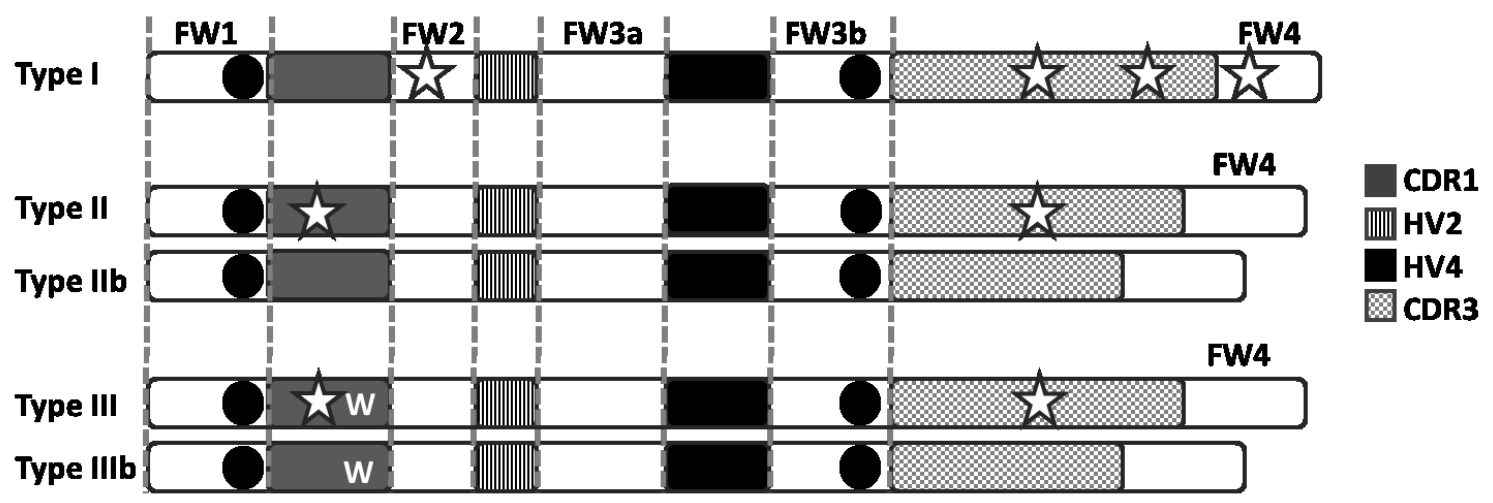

Figure 2. Representation of VNAR types. Black filler circles depict canonical cysteine residues; open stars depict non-canonical cysteine residues; FW, framework. The Type I domain often has a slightly larger molecular weight than other VNAR sub-types reflecting the extended CDR3 region which can consist of up to 35 amino acids. 
VNARs however are not limited to binding into canyons or clefts. Kovalenko and colleagues crystallized a Type IIb domain that was raised against HSA in a spiny dogfish [31,34]. This domain lacks the non-canonical CDR-framework cysteine and resultantly has a more flexible structure. The interface between the domain E06 and HSA was more planar with the CDR and HV still constituting the majority of the paratope but with additional contribution from framework residues. Interestingly, this increased structural flexibility compared with other VNAR isotypes, allowed the addition, through gene cloning and recombinant protein expression, of $\mathrm{N}$ and $\mathrm{C}$ terminal fusions without any loss of functional activity of the core E06 domain (see Table 1).

Taken together, the structural diversity of VNAR domains maximizes their potential to bind unique epitopes, and may reflect a natural pre-disposition to generate neutralizing binders that recognize enzyme or receptor active sites. Therefore using VNAR as the starting point for drug discovery should expand the repertoire of druggable protein targets and potentially increase the potency of biological outcomes.

Table 1. Comparison of the species specific affinity (via BIAcore) and in vivo half-life extension (after a single i.v. administration) of fusion proteins consisting at their core of albumin binding domains derived from either camel or shark.

\begin{tabular}{ccccc}
\hline \multirow{2}{*}{$\begin{array}{c}\text { Domain Type and } \\
\text { Orientations }\end{array}$} & \multicolumn{2}{c}{ Mean KD Albumin $(\mathbf{n M})$} & \multicolumn{2}{c}{ Mean t $\mathbf{t}_{\mathbf{1} 2}$ (days) } \\
\cline { 2 - 5 } Couse & Monkey & Mouse & Monkey \\
\hline Camel VHH & & & & \\
ABVHH & 73 & N/A & 0.5 & N/A \\
VHH-ABVHH & 470 & N/A & 0.6 & 4.8 \\
VHH-ABVHH-VHH & 180 & N/A & 1.4 & N/A \\
Shark VNAR & & & & \\
ABVNAR & 0.7 & 0.2 & N/A & N/A \\
VNAR-ABVNAR & 2.5 & 0.9 & 1.4 & 6.7 \\
VNAR-ABVNAR-VNAR & 4.0 & 1.0 & & \\
\hline
\end{tabular}

Single Domain Formats: ABVHH and ABVNAR refer to camel and shark albumin binding domains respectively. The albumin binding domains are fused to either camel (VHH) or shark (VNAR) domains at either the $\mathrm{N}$ or $\mathrm{N}$ and $\mathrm{C}$ termini of the expressed protein. N/A: Comparable data was not available.

\subsubsection{Molecular Malleability}

The diversity of the naïve VNAR repertoire is dominated by CDR3 and is achieved through V(D)J recombination. In nurse shark a combination of three $\mathrm{D}$ regions with additional junctional variation via the N-region and P-nucleotide additions, results in significant sequence diversity $[48,49]$. The exception to this molecular route to diversity are the Type III VNARs which have a fused D region significantly limiting variation. The purpose of this Type III VNAR is believed to provide early protection to young animals against a common pathogen, a hypothesis supported by their expression patterns observed only in young animals [49].

As our broad understanding of VNAR biology and structure has matured, so our ability to recreate synthetic VNAR diversity in the laboratory has become more refined. Both semi and fully synthetic VNAR binding libraries have been constructed with varying degrees of success. Crucial to this approach is the ability to isolate target-specific VNAR domains from diverse, combinatorial repertoires and both 
phage and yeast display have been used successfully as robust and reliable selection platforms [20,30,50]. Nuttall et al. built the first reported semi-synthetic VNAR phage libraries from wobbegong sharks, isolating a number of domains against AMA-1, Tom70 and Gingipain K protease [23,24,51,52]. These domains provided further valuable insights into the structure or topology of VNAR binding sites and were used successfully as the basis for random and rational in vitro maturation to deliver increased binding affinities to target antigen [53-55]. In an extension of this approach Shao et al. were able to take a VNAR specific for lysozyme and through a process of mutation and reselection convert the this VNAR into a binding domain that was specific for a second and very different protein Leptin [50]. In most of these published studies, the primary focus for creating library diversity has been through the comprehensive mutagenesis of CDR3, as it is this loop that is believed (mimicking the natural process for naïve VNAR repertoire diversification) to play a central role in antigen recognition and binding $[23,24,51,52]$. However the HV loops can also contribute not only to increased affinity of binding through increased interface interactions but can also bind target independently. The importance of HV loop binding was demonstrated recently by Zielonka and colleagues who built an HV2 yeast display library from an EpCAM specific VNAR and selected domains that also bound a second protein target [56]. This novel example of bi-specificity was achieved by utilizing different loops within a single VNAR binding site structure.

In another separate study the interrogation of the potential amino acid positions that were available for mutagenesis within an anti-HEL Type I domain, 5A7, was achieved using ribosome display and a functional selection strategy based on antigen binding [57]. This data set provided further valuable insights into the plasticity of this domain by revealing residues and regions that could be mutated without affecting functionality. In addition, and following comparison to other VNAR from other several other species, the authors were able to pinpoint hallmark residues which form the core of the domain structure, affording them their remarkable biophysical properties that included stability in a range of non-physiological conditions.

Due to their single domain architecture, re-formatting of VNAR is simple compared to other more complex structures, enabling domains to be tailored for a particular purpose. These novel structures include "plug-and-play" molecular fusions consisting of two or more VNAR domains linked in tandem [31,58]. This permits the expression of functional bi and tri-specific formats capable of binding more than one target or increasing affinity to a single target through avidity (or both). Other formats such as Fc fusions have been constructed, expressed and purified. These VNAR-antibody hybrids have a long serum half-life, can recruit for cell killing, but are only half the molecular weight of mAbs and retain the novel binding characteristics achieved through their unique paratope recognition [34].

\subsubsection{Remarkable Stability}

Whether it is a consequence of the harsh plasma environment of sharks or the small compact, disulphide linked structural features of VNARs or a combination of both, these domains are incredibly robust. VNARs can be heated $\left(>80^{\circ} \mathrm{C}\right)$ for up to an hour and still exhibit the ability to bind target when returned to physiological conditions $[20,25,59]$. Such studies exemplify the ability of these domains to not only resist denaturation but to rapidly refold into a functional conformation upon removal of the heat challenge. In related studies, they can be incubated at room temperature or at $37{ }^{\circ} \mathrm{C}$ for up to a month 
with negligible detriment to function, demonstrating the potential for long shelf-life stability or for sustained therapeutic delivery from depot or implants. Other challenges including low $\mathrm{pH}$ and protease resistance have been explored to confirm the potential of these domains to survive for extended periods in the gut. Stability is especially impressive when compared, in functional assays, with the stability of other binding site structures such as antibody fragments [60].

Inherent stability not only increases the therapeutic potential and delivery options of these domains but also makes them ideal for reagent or diagnostic development. Griffiths showed that nitrocellulose immobilized VNARs were significantly more stable than mAbs when incubated at $45{ }^{\circ} \mathrm{C}$ for up to four weeks [60].

\section{VNARs in Development}

\subsection{NDure ${ }^{\mathrm{TM}}$ - The First Clinical Candidate}

The first VNAR domain to be developed for clinical use is an HSA-specific domain (NDure ${ }^{\mathrm{TM}}$ ) isolated from an immunized spiny dogfish library. Originally called E06 it was then humanized for use in man [31,34]. By binding to HSA NDure ${ }^{\mathrm{TM}}$ is not a therapeutic domain in its own right but it is a critical tool required to improve the efficacy of other fusion protein-products, by increasing their serum half-life and subsequently their therapeutic exposure time. Binding to albumin as a means of half-life extension is a validated mechanism of action and employs the same FcRN receptor system that gives antibodies their extended plasma circulation times [61-64]. Camel VHH domains have also been isolated to serum albumins and successfully exploited to extend the serum half-life in vivo of a number of fusion partner proteins including other VHH domains. Published data [65] allows some degree of comparison to be made between the performance of an anti-albumin VHH domain and the E06 VNAR domain including (Table 1): species cross-reactivity, over all affinity, domain fusion flexibility and the resulting half-life extension of partner proteins. A good level of functional affinity is demonstrated by both domains classes with VNAR, at least in this comparison, showing a slight but significant improvement in terms of affinity and half-life extension over the equivalent VHH domain.

\subsubsection{E06-Fit for Purpose}

There are a number of criteria that need to be fulfilled to develop an optimal domain capable of extending the serum half-life of fused partner molecules: (1) selectivity for albumin with cross-species specificity; (2) binding to an epitope of albumin that does not directly or indirectly effect FcRN binding; (3) binding at both neutral and acidic $\mathrm{pH}$ to remain in complex with albumin during intracellular recycling; (4) tolerate $\mathrm{N}$ and $\mathrm{C}$-terminal (or both together) fusions of a range of proteins including: peptides, antibody fragments and other VNAR domains (5) have sufficient sequence plasticity to be humanized whilst retaining affinity and specificity for target.

All of these considerations were engineered into the selection and screening campaign that resulted in the isolation of the lead domain, E06. With an inherently high affinity for human albumin (pM), E06 also binds mouse, rat and monkey albumins (Table 1) enabling pre-clinical models to be conducted with the same candidate molecule. Being a Type IIb domain, it has a flexible structure which tolerates $\mathrm{N}$-terminal, or C-terminal or both termini fusion to partner domains without sacrificing function. Indeed, 
E06 has not only been fused to other partner VNAR domains (both therapeutic and model) but a number of other relevant proteins and peptides (Figure 3) whilst still retaining albumin binding and importantly the function of the fusion partner.

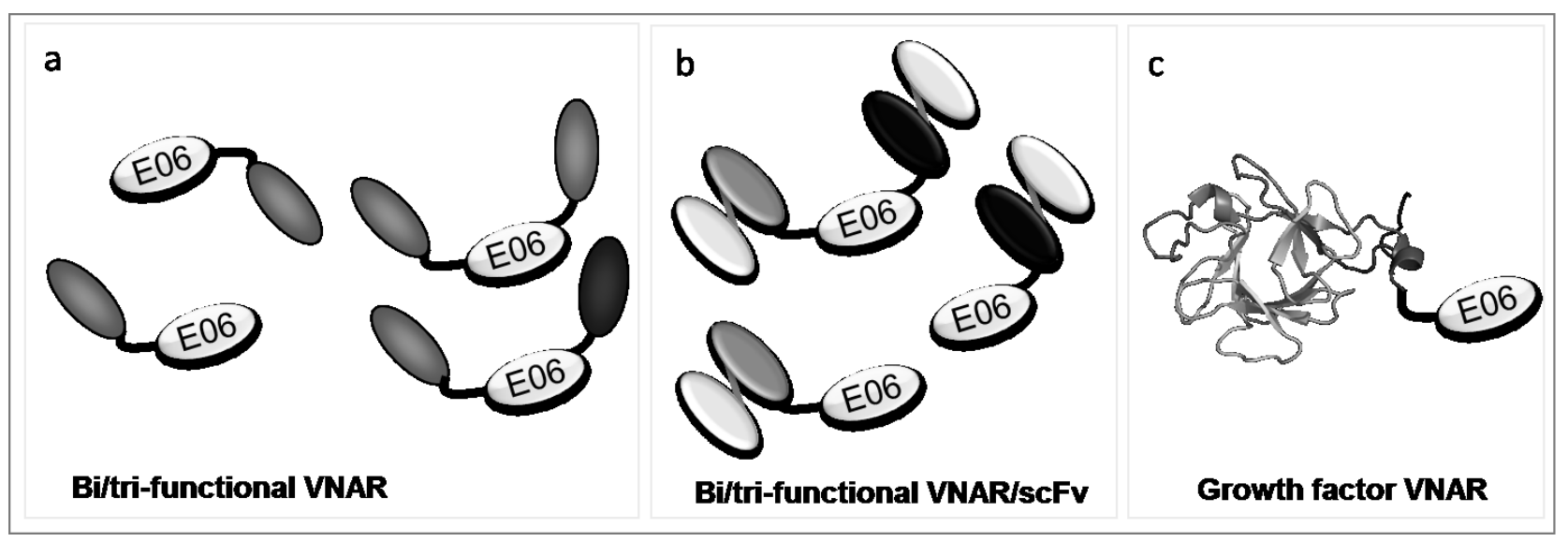

Figure 3. Validated formats for half-life extension (a) N-terminal, C-terminal and dual terminal fusions of E06 with partner VNAR domains; (b) N-terminal, C-terminal and dual terminal fusions of E06 with partner scFv domains; (c) E06 growth factor fusion product.

The efficacy of E06, in all three fusion formats shown in Figure 3a (dimer and trimer) were assessed in PK models in three different species (mouse, rat and monkey). An extension of the monkey study also confirmed that this product could be administered subcutaneously as well as intravenously with both routes of administration leading to rapid bioavailability and half-life extension values of over 6 days. Extrapolation of these data, predicts that the half-life of an E06 fusion in man would be approximately 19 days, mirroring that of human albumin [44].

\subsubsection{Becoming Human - the Creation of NDure ${ }^{\mathrm{TM}}$}

From the first antibodies in the clinic through to those entering trials today, there has been a trend towards becoming "human". Different approaches such as complementary determining region (CDR) grafting, framework/germline-homology scaffolds and molecular re-surfacing have all been adopted to reduce potential immunogenic responses from non-human (and sometimes even human) therapeutic biologics following administration [66-69]. According to Reichert (2012), of the 28 marketed mAbs in Europe and the US, the majority (36\%) were humanized, exemplifying the success and subsequent approval of this process [70]. Of these, probably the best known are the blockbuster mAbs, Trastuzumab (Herceptin) and Bevacizumab (Avastin) each of which generated in excess of \$6 billion in revenues in 2012.

As the origin of VNAR domains are non-human and indeed not even mammalian, the question of humanization becomes more important. E06 was successfully humanized using a method of structural homology modelling to human heavy chains [34]. By aligning and choosing not only the most similar framework but also the most biophysically attractive (for the purposes of clinical development), the team successfully humanized both Type I and Type IIb domains, 5A7 and E06 respectively, and demonstrated retention of antigen binding (Figure 4). In the literature, to distinguish humanized VNAR domains from their parental VNAR structures, they are now often referred to as soloMERs ${ }^{\mathrm{TM}}$. 


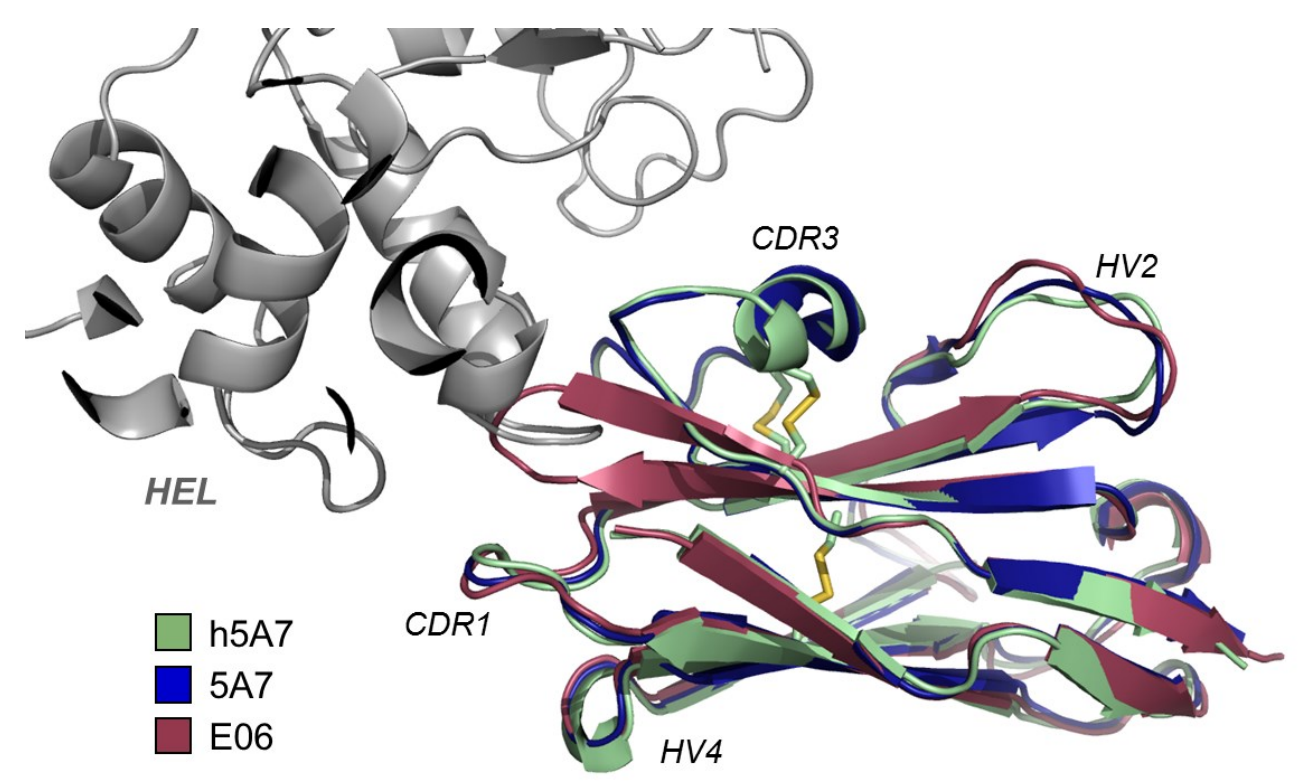

Figure 4. Humanized soloMER ${ }^{\mathrm{TM}} 5 \mathrm{~A} 7$ (h5A7) and E06 superimposed onto a structural model based on 5A7 in complex with HEL [46].

The humanized (or soloMER ${ }^{\mathrm{TM}}$ ) version of E06 is known as NDure ${ }^{\mathrm{TM}}$ and has shown negligible antigenicity in ex vivo models, retained binding as a fusion product, extension of half-life in vivo and high levels of stability and solubility. It has also been shown to be highly amenable to site-specific chemical conjugation (manuscript in preparation).

\subsection{Other Reported VNAR Domains}

A number of other VNAR domains specific for a range of antigen targets have now been isolated and characterized from both immunized and synthetic platforms, and from a number of different species including horn shark and bamboo shark (Table 2).

The majority of the earliest antigen-specific VNAR domains came from semi or fully synthetic libraries and were typically used as simple reagent molecules, designed to detect and identify target rather than as possible treatments for disease [25,26,29,30,33,51,52,71,72]. More recently, with the development of better platforms for VNAR isolation, including immunization, the potential for these domains in a therapeutic setting was considered for the first time. The original VNAR to be studied in pre-clinical models was E06 which clearly demonstrated the stability and utility of these domains in vivo with half-life extension of fusion partner proteins close to the serum albumin half-life of the animal models used in this study (Table 1).

A second VNAR domain that originated from an immunized animal has also been assessed in pre-clinical models. The anti-TNF $\alpha$ domain, T43, was originally isolated from a horn shark immunized with the human version of the cytokine [33]. This domain was assessed in a murine model of endotoxic shock and showed a measurable but modest level of protection compared to the control [73]. There is a huge market for anti-TNF $\alpha$ products but any new player in this space will have to demonstrate substantial improvement or differentiation over the competition. It will be interesting to see if this domain or other anti-TNF domains currently in development, and derived originally from nurse shark immune libraries, can progress towards clinical use. 
Table 2. VNARs reported in the literature.

\begin{tabular}{clc}
\hline \multicolumn{1}{c}{ Purpose } & \multicolumn{1}{c}{ Target } & Reference \\
\hline \multirow{4}{*}{ Therapeutic } & EpCAM & {$[30]$} \\
& VHSV & {$[29]$} \\
& TNF-alpha & {$[33]$} \\
& HBeAg of HBV & {$[72]$} \\
& Ebola virus & {$[71]$} \\
& Cholera toxin & {$[25]$} \\
& Staphylococcal enterotoxin B & {$[26]$} \\
Diagnostic/Reagent & Ricin & {$[26]$} \\
& Botulinum toxin & {$[26]$} \\
& AMA1 & {$[52]$} \\
& Tom70 & {$[51]$} \\
\hline
\end{tabular}

\section{Conclusions}

From its ancient beginnings, VNARs or at least soloMERs ${ }^{\mathrm{TM}}$ may soon find applications at the heart of modern medical practice. The extensive research undertaken to unravel the biological origins of this domain has not only laid the foundation for our understanding of the primordial adaptive immunity but has also revealed a potential new therapeutic technology platform. It is its natural ability to bind target with high affinity and specificity that sets this domain a part with the potential to become the basis of a powerful, novel and differentiated drug-discovery engine. VNARs/soloMERs ${ }^{\mathrm{TM}}$ inherent small size, stability and solubility all contribute to the criteria required for a viable development path. Amongst this plethora of drug-like attributes, the ability or natural pre-disposition to target novel epitopes should not be underestimated. The tackling of new disease molecule classes could now be possible (e.g., GPCRs or Ion channels) and improvements upon existing biologics achieved, by neutralization of different parts of existing and validated protein drug targets, to deliver increases in therapeutic potency.

The simple single chain structure of this domains lends itself perfectly to multiple reformatting options, enabling products to be properly tailored for clinical need (systemic or site specific delivery). Therefore, the smallest binding sites in the natural kingdom, could one day become the next big protein drug class and play its part in the on-going biologics revolution. Only time will tell.

\section{Acknowledgments}

This work was supported by Scottish Enterprise (SE) and the Biotechnology and Biological Sciences Research Council (BBSRC).

\section{Author Contributions}

The authors contributed equally to this manuscript.

\section{Conflicts of Interest}

The authors declare no conflict of interest. 


\section{References}

1. Papermaster, B.W.; Condie, R.M.; Finstad, J.; Good, R.A. Evolution of the Immune Response. I. the Phylogenetic Development of Adaptive Immunologic Responsiveness in Vertebrates. J. Exp. Med. 1964, 119, 105-130.

2. Parton, A.; Bayne, C.J.; Barnes, D.W. Analysis and functional annotation of expressed sequence tags from in vitro cell lines of elasmobranchs: Spiny dogfish shark (Squalus acanthias) and little skate (Leucoraja erinacea). Comp. Biochem. Physiol. Part D. Genomics Proteomics 2010, 5, 199-206.

3. Marchalonis, J.J.; Schluter, S.F.; Bernstein, R.M.; Shen, S.; Edmundson, A.B. Phylogenetic emergence and molecular evolution of the immunoglobulin family. Adv. Immunol. 1998, 70, 417-506.

4. Flajnik, M.F.; Rumfelt, L.L. The immune system of cartilaginous fish. Curr. Top. Microbiol. Immunol. 2000, 248, 249-270.

5. Fange, R.; Pulsford, A. Structural studies on lymphomyeloid tissues of the dogfish, Scyliorhinus canicula L. Cell Tissue Res. 1983, 230, 337-351.

6. Rumfelt, L.L.; Avila, D.; Diaz, M.; Bartl, S.; McKinney, E.C.; Flajnik, M.F. A shark antibody heavy chain encoded by a nonsomatically rearranged VDJ is preferentially expressed in early development and is convergent with mammalian IgG. Proc. Natl. Acad. Sci. USA 2001, 98, 1775-1780.

7. Miracle, A.L.; Anderson, M.K.; Litman, R.T.; Walsh, C.J.; Luer, C.A.; Rothenberg, E.V.; Litman, G.W. Complex expression patterns of lymphocyte-specific genes during the development of cartilaginous fish implicate unique lymphoid tissues in generating an immune repertoire. Int. Immunol. 2001, 13, 567-580.

8. Rumfelt, L.L.; McKinney, E.C.; Taylor, E.; Flajnik, M.F. The development of primary and secondary lymphoid tissues in the nurse shark Ginglymostoma cirratum: B-cell zones precede dendritic cell immigration and T-cell zone formation during ontogeny of the spleen. Scand. J. Immunol. 2002, 56, 130-148.

9. Clem, L.W.; De Boutard, F.; Sigel, M.M. Phylogeny of immunoglobulin structure and function - II. Immunoglobulins of the nurse shark. J. Immunol. 1967, 99, 1226-1235.

10. Clem, L.W.; Small, P.A., Jr. Phylogeny of immunoglobulin structure and function. I. Immunoglobulins of the lemon shark. J. Exp. Med. 1967, 125, 893-920.

11. Marchalonis, J.; Edelman, G.M. Phylogenetic origins of antibody structure. I. Multichain structure of immunoglobulins in the smooth dogfish (Mustelus canis). J. Exp. Med. 1965, 122, 601-618.

12. Marchalonis, J.; Edelman, G.M. Polypeptide chains of immunoglobulins from the smooth dogfish (Mustelus canis). Science 1966, 154, 1567-1568.

13. Morrow, W.; Harris, J.E; Pulsford, A. Immunological responses of the dogfish (Scyliorhinus canicula L.) to cellular antigens. Acta Zoologica (Stockh.) 1982, 63, 153-159.

14. Morrow, W.J.W.; Harris, J.E.; Davies, D.; Pulsford, A.A. Isolation and partial characterization of dogfish (Scyliorhinus canicula L) antibody. J. Mar. Biol. Ass. UK 1983, 63, 409-418.

15. Rumfelt, L.L.; Diaz, M.; Lohr, R.L.; Mochon, E.; Flajnik, M.F. Unprecedented multiplicity of Ig transmembrane and secretory mRNA forms in the cartilaginous fish. J. Immunol. 2004, 173, 1129-1139.

16. Rumfelt, L.L.; Lohr, R.L.; Dooley, H.; Flajnik, M.F. Diversity and repertoire of IgW and IgM VH families in the newborn nurse shark. BMC Immunol. 2004, 5, 8-22. 
17. Greenberg, A.S.; Avila, D.; Hughes, M.; Hughes, A.; McKinney, E.C.; Flajnik, M.F. A new antigen receptor gene family that undergoes rearrangement and extensive somatic diversification in sharks. Nature 1995, 374, 168-173.

18. Dooley, H.; Stanfield, R.L.; Brady, R.A.; Flajnik, M.F. First molecular and biochemical analysis of in vivo affinity maturation in an ectothermic vertebrate. Proc. Natl. Acad. Sci. USA 2006, 103, 1846-1851.

19. Feige, M.J.; Grawert, M.A.; Marcinowski, M.; Hennig, J.; Behnke, J.; Auslander, D.; Herold, E.M.; Peschek, J.; Castro, C.D.; Flajnik, M.; et al. The structural analysis of shark IgNAR antibodies reveals evolutionary principles of immunoglobulins. Proc. Natl. Acad. Sci. USA 2014, 111, 8155-8160.

20. Dooley, H.; Flajnik, M.F.; Porter, A.J. Selection and characterization of naturally occurring singledomain (IgNAR) antibody fragments from immunized sharks by phage display. Mol. Immunol. 2003, 40, 25-33.

21. Dooley, H.; Flajnik, M.F. Shark immunity bites back: affinity maturation and memory response in the nurse shark, Ginglymostoma cirratum. Eur. J. Immunol. 2005, 35, 936-945.

22. Diaz, M.; Velez, J.; Singh, M.; Cerny, J.; Flajnik, M.F. Mutational pattern of the nurse shark antigen receptor gene (NAR) is similar to that of mammalian Ig genes and to spontaneous mutations in evolution: The translesion synthesis model of somatic hypermutation. Int. Immunol. 1999, 11, 825-833.

23. Nuttall, S.D.; Krishnan, U.V.; Hattarki, M.; De Gori, R.; Irving, R.A.; Hudson, P.J. Isolation of the new antigen receptor from wobbegong sharks, and use as a scaffold for the display of protein loop libraries. Mol. Immunol. 2001, 38, 313-326.

24. Nuttall, S.D.; Krishnan, U.V.; Doughty, L.; Nathanielsz, A.; Ally, N.; Pike, R.N.; Hudson, P.J.; Kortt, A.A.; Irving, R.A. A naturally occurring NAR variable domain binds the Kgp protease from Porphyromonas gingivalis. FEBS Lett. 2002, 516, 80-86.

25. Liu, J.L.; Anderson, G.P.; Delehanty, J.B.; Baumann, R.; Hayhurst, A.; Goldman, E.R. Selection of cholera toxin specific IgNAR single-domain antibodies from a naive shark library. Mol. Immunol. 2007, 44, 1775-1783.

26. Liu, J.L.; Anderson, G.P.; Goldman, E.R. Isolation of anti-toxin single domain antibodies from a semi-synthetic spiny dogfish shark display library. BMC Biotechnol. 2007, 7, 78-88.

27. Crouch, K.; Smith, L.E.; Williams, R.; Cao, W.; Lee, M.; Jensen, A.; Dooley, H. Humoral immune response of the small-spotted catshark, Scyliorhinus canicula. Fish Shellfish Immunol. 2013, 34, $1158-1169$.

28. Ohtani, M.; Hikima, J.; Jung, T.S.; Kondo, H.; Hirono, I.; Aoki, T. Construction of an artificially randomized IgNAR phage display library: Screening of variable regions that bind to hen egg white lysozyme. Mar. Biotechnol. (NY) 2013, 15, 56-62.

29. Ohtani, M.; Hikima, J.; Jung, T.S.; Kondo, H.; Hirono, I.; Takeyama, H.; Aoki, T. Variable domain antibodies specific for viral hemorrhagic septicemia virus (VHSV) selected from a randomized IgNAR phage display library. Fish Shellfish Immunol. 2013, 34, 724-728.

30. Zielonka, S.; Weber, N.; Becker, S.; Doerner, A.; Christmann, A.; Christmann, C.; Uth, C.; Fritz, J.; Schafer, E.; Steinmann, B.; et al. Shark Attack: high affinity binding proteins derived from shark vNAR domains by stepwise in vitro affinity maturation. J. Biotechnol. 2014, 191, 236-245. 
31. Müller, M.R.; Saunders, K.; Grace, C.; Jin, M.; Piche-Nicholas, N.; Steven, J.; O'Dwyer, R.; Wu, L.; Khetemenee, L.; Vugmeyster, Y.; et al. Improving the pharmacokinetic properties of biologics by fusion to an anti-HSA shark VNAR domain. MAbs 2012, 4, 673-685.

32. Müller, M.R.; O'Dwyer, R.; Kovaleva, M.; Rudkin, F.; Dooley, H.; Barelle, C.J. Generation and isolation of target-specific single-domain antibodies from shark immune repertoires. Methods Mol. Biol. 2012, 907, 177-194.

33. Camacho-Villegas, T.; Mata-Gonzalez, T.; Paniagua-Solis, J.; Sanchez, E.; Licea, A. Human TNF cytokine neutralization with a vNAR from Heterodontus francisci shark: a potential therapeutic use. MAbs 2013, 5, 80-85.

34. Kovalenko, O.V.; Olland, A.; Piche-Nicholas, N.; Godbole, A.; King, D.; Svenson, K.; Calabro, V.; Muller, M.R.; Barelle, C.J.; Somers, W.; et al. Atypical Antigen Recognition Mode of a Shark IgNAR Variable Domain Characterized by Humanization and Structural Analysis. J. Biol. Chem. 2013, 288, 17408-17419.

35. Dooley, H.; Flajnik, M.F. Antibody repertoire development in cartilaginous fish. Dev. Comp. Immunol. 2006, 30, 43-56.

36. Flajnik, M.F. Immunogenetics: Alternative strategies in adaptive immunity and the rise of comparative immunogenomics. Curr. Opin. Immunol. 2007, 19, 522-525.

37. Criscitiello, M.F.; Saltis, M.; Flajnik, M.F. An evolutionarily mobile antigen receptor variable region gene: Doubly rearranging NAR-TcR genes in sharks. Proc. Natl. Acad. Sci. USA 2006, 103, 5036-5041.

38. Hinds, K.R.; Litman, G.W. Major reorganization of immunoglobulin VH segmental elements during vertebrate evolution. Nature 1986, 320, 546-549.

39. Litman, G.W.; Anderson, M.K.; Rast, J.P. Evolution of antigen binding receptors. Annu. Rev. Immunol. 1999, 17, 109-147.

40. Greenberg, A.S.; Hughes, A.L.; Guo, J.; Avila, D.; McKinney, E.C.; Flajnik, M.F. A novel "chimeric" antibody class in cartilaginous fish: IgM may not be the primordial immunoglobulin. Eur. J. Immunol. 1996, 26, 1123-1129.

41. Venkatesh, B.; Lee, A.P.; Ravi, V.; Maurya, A.K.; Lian, M.M.; Swann, J.B.; Ohta, Y.; Flajnik, M.F.; Sutoh, Y.; Kasahara, M.; et al. Elephant shark genome provides unique insights into gnathostome evolution. Nature 2014, 505, 174-179.

42. Muyldermans, S. Nanobodies: Natural Single-Domain Antibodies. Annu. Rev. Biochem. 2013, 82, 775-797.

43. Flajnik, M.F.; Deschacht, N.; Muyldermans, S. A case of convergence: Why did a simple alternative to canonical antibodies arise in sharks and camels? PLoS Biol. 2011, 9, 1-5.

44. Kovaleva, M.; Ferguson, L.; Steven, J.; Porter, A.; Barelle, C. Shark variable new antigen receptor biologics - a novel technology platform for therapeutic drug development. Expert Opin. Biol. Ther. 2014, 14, 1527-1539.

45. Zielonka, S.; Empting, M.; Grzeschik, J.; Konning, D.; Barelle, C.J.; Kolmar, H. Structural insights and biomedical potential of IgNAR scaffolds from sharks. MAbs 2015, 7, 15-25.

46. Stanfield, R.L.; Dooley, H.; Flajnik, M.F.; Wilson, I.A. Crystal structure of a shark single-domain antibody V region in complex with lysozyme. Science 2004, 305, 1770-1773. 
47. Streltsov, V.A.; Carmichael, J.A.; Nuttall, S.D. Structure of a shark IgNAR antibody variable domain and modeling of an early-developmental isotype. Protein Sci. 2005, 14, 2901-2909.

48. Diaz, M.; Greenberg, A.S.; Flajnik, M.F. Somatic hypermutation of the new antigen receptor gene (NAR) in the nurse shark does not generate the repertoire: possible role in antigen-driven reactions in the absence of germinal centers. Proc. Natl. Acad. Sci. USA 1998, 95, 14343-14348.

49. Diaz, M.; Stanfield, R.L.; Greenberg, A.S.; Flajnik, M.F. Structural analysis, selection, and ontogeny of the shark new antigen receptor (IgNAR): Identification of a new locus preferentially expressed in early development. Immunogenetics 2002, 54, 501-512.

50. Shao, C.Y.; Secombes, C.J.; Porter, A.J. Rapid isolation of IgNAR variable single-domain antibody fragments from a shark synthetic library. Mol. Immunol. 2007, 44, 656-665.

51. Nuttall, S.D.; Krishnan, U.V.; Doughty, L.; Pearson, K.; Ryan, M.T.; Hoogenraad, N.J.; Hattarki, M.; Carmichael, J.A.; Irving, R.A.; Hudson, P.J. Isolation and characterization of an IgNAR variable domain specific for the human mitochondrial translocase receptor Tom70. Eur. J. Biochem. 2003, 270, 3543-3554.

52. Nuttall, S.D.; Humberstone, K.S.; Krishnan, U.V.; Carmichael, J.A.; Doughty, L.; Hattarki, M.; Coley, A.M.; Casey, J.L.; Anders, R.F.; Foley, M.; et al. Selection and affinity maturation of IgNAR variable domains targeting Plasmodium falciparum AMA1. Proteins 2004, 55, 187-197.

53. Streltsov, V.A.; Varghese, J.N.; Carmichael, J.A.; Irving, R.A.; Hudson, P.J.; Nuttall, S.D. Structural evidence for evolution of shark Ig new antigen receptor variable domain antibodies from a cell-surface receptor. Proc. Natl. Acad. Sci. USA 2004, 101, 12444-12449.

54. Kopsidas, G.; Roberts, A.S.; Coia, G.; Streltsov, V.A.; Nuttall, S.D. In vitro improvement of a shark IgNAR antibody by Qbeta replicase mutation and ribosome display mimics in vivo affinity maturation. Immunol. Lett. 2006, 107, 163-168.

55. Henderson, K.A.; Streltsov, V.A.; Coley, A.M.; Dolezal, O.; Hudson, P.J.; Batchelor, A.H.; Gupta, A.; Bai, T.; Murphy, V.J.; Anders, R.F.; et al. Structure of an IgNAR-AMA1 complex: targeting a conserved hydrophobic cleft broadens malarial strain recognition. Structure 2007, 15, 1452-1466.

56. Zielonka, S.; Empting, M.; Konning, D.; Grzeschik, J.; Krah, S.; Becker, S.; Dickgiesser, S.; Kolmar, H. The Shark Strikes Twice: Hypervariable Loop 2 of Shark IgNAR Antibody Variable Domains and Its Potential to Function as an Autonomous Paratope. Mar. Biotechnol. (NY) 2015, 17, 386-392.

57. Fennell, B.J.; Darmanin-Sheehan, A.; Hufton, S.E.; Calabro, V.; Wu, L.; Muller, M.R.; Cao, W.; Gill, D.; Cunningham, O.; Finlay, W.J. Dissection of the IgNAR V domain: molecular scanning and orthologue database mining define novel IgNAR hallmarks and affinity maturation mechanisms. J. Mol. Biol. 2010, 400, 155-170.

58. Simmons, D.P.; Abregu, F.A.; Krishnan, U.V.; Proll, D.F.; Streltsov, V.A.; Doughty, L.; Hattarki, M.K.; Nuttall, S.D. Dimerisation strategies for shark IgNAR single domain antibody fragments. J. Immunol. Methods 2006, 315, 171-184.

59. Liu, J.L.; Zabetakis, D.; Brown, J.C.; Anderson, G.P.; Goldman, E.R. Thermal stability and refolding capability of shark derived single domain antibodies. Mol. Immunol. 2014, 59, 194-199.

60. Griffiths, K.; Dolezal, O.; Parisi, K.; Angerosa, J.; Dogovski, C.; Barraclough, M.; Sanalla, A.; Casey, J.; Gonzalez, I.; Perugini, M.; et al. Shark variable new antigen receptor (VNAR) single domain antibody fragments: Stability and diagnostic applications. Antibodies 2013, 2, 66-88. 
61. Markussen, J.; Havelund, S.; Kurtzhals, P.; Andersen, A.S.; Halstrom, J.; Hasselager, E.; Larsen, U.D.; Ribel, U.; Schaffer, L.; Vad, K.; et al. Soluble, fatty acid acylated insulins bind to albumin and show protracted action in pigs. Diabetologia 1996, 39, 281-288.

62. Makrides, S.C.; Nygren, P.A.; Andrews, B.; Ford, P.J.; Evans, K.S.; Hayman, E.G.; Adari, H.; Uhlen, M.; Toth, C.A. Extended in vivo half-life of human soluble complement receptor type 1 fused to a serum albumin-binding receptor. J. Pharmacol. Exp. Ther. 1996, 277, 534-542.

63. Smith, B.J.; Popplewell, A.; Athwal, D.; Chapman, A.P.; Heywood, S.; West, S.M.; Carrington, B.; Nesbitt, A.; Lawson, A.D.; Antoniw, P.; et al. Prolonged in vivo residence times of antibody fragments associated with albumin. Bioconjug. Chem. 2001, 12, 750-756.

64. Stork, R.; Muller, D.; Kontermann, R.E. A novel tri-functional antibody fusion protein with improved pharmacokinetic properties generated by fusing a bispecific single-chain diabody with an albumin-binding domain from streptococcal protein G. Protein Eng. Des. Sel. 2007, 20, 569-576.

65. Van Roy, M.; Ververken, C.; Beirnaert, E.; Hoefman, S.; Kolkman, J.; Vierboom, M.; Breedveld, E.; 't Hart, B.; Poelmans, S.; Bontinck, L.; et al. The preclinical pharmacology of the high affinity anti-IL-6R Nanobody(R) ALX-0061 supports its clinical development in rheumatoid arthritis. Arthritis Res. Ther. 2015, 17, 135-150.

66. Padlan, E.A. A possible procedure for reducing the immunogenicity of antibody variable domains while preserving their ligand-binding properties. Mol. Immunol. 1991, 28, 489-498.

67. Pelat, T.; Bedouelle, H.; Rees, A.R.; Crennell, S.J.; Lefranc, M.P.; Thullier, P. Germline humanization of a non-human primate antibody that neutralizes the anthrax toxin, by in vitro and in silico engineering. J. Mol. Biol. 2008, 384, 1400-1407.

68. Robert, R.; Streltsov, V.A.; Newman, J.; Pearce, L.A.; Wark, K.L.; Dolezal, O. Germline humanization of a murine Abeta antibody and crystal structure of the humanized recombinant Fab fragment. Protein Sci. 2010, 19, 299-308.

69. Cheung, N.K.; Guo, H.; Hu, J.; Tassev, D.V.; Cheung, I.Y. Humanizing murine IgG3 anti-GD2 antibody m3F8 substantially improves antibody-dependent cell-mediated cytotoxicity while retaining targeting in vivo. Oncoimmunology 2012, 1, 477-486.

70. Reichert, J.M. Marketed therapeutic antibodies compendium. MAbs 2012, 4, 413-415.

71. Goodchild, S.A.; Dooley, H.; Schoepp, R.J.; Flajnik, M.; Lonsdale, S.G. Isolation and characterisation of Ebolavirus-specific recombinant antibody fragments from murine and shark immune libraries. Mol. Immunol. 2011, 48, 2027-2037.

72. Walsh, R.; Nuttall, S.; Revill, P.; Colledge, D.; Cabuang, L.; Soppe, S.; Dolezal, O.; Griffiths, K.; Bartholomeusz, A.; Locarnini, S. Targeting the hepatitis B virus precore antigen with a novel IgNAR single variable domain intrabody. Virology 2011, 411, 132-141.

73. Bojalil, R.; Mata-Gonzalez, M.T.; Sanchez-Munoz, F.; Yee, Y.; Argueta, I.; Bolanos, L.; AmezcuaGuerra, L.M.; Camacho-Villegas, T.A.; Sanchez-Castrejon, E.; Garcia-Ubbelohde, W.J.; et al. Antitumor necrosis factor VNAR single domains reduce lethality and regulate underlying inflammatory response in a murine model of endotoxic shock. BMC Immunol. 2013, 14, 17.

(C) 2015 by the authors; licensee MDPI, Basel, Switzerland. This article is an open access article distributed under the terms and conditions of the Creative Commons Attribution license (http://creativecommons.org/licenses/by/4.0/). 